\title{
Assembly and Reconstruction of Cytotoxic Bacterial Protein Assemblies in Lipid Vesicles
}

\section{Cristina Fernández}

cfernandez@cib.csic.es

Germán Rivas

\section{Rafael Giraldo}

\section{Mercedes Jiménez}

enoe@cib.csic.es

CIB-CSIC, Madrid, Spain

${ }^{1}$ Corresponding authors:(Please, note that corresponding authors are Cristina Fernández and Mercedes Jiménez. As the corresponding authors are indicated with a superscript (1), colon should be deleted.)

Abstract

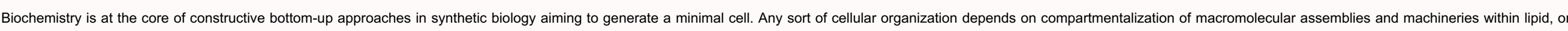

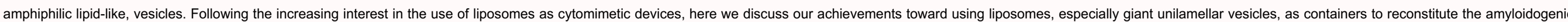

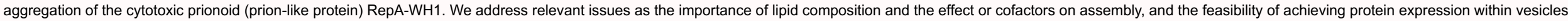

Abbreviations

$\mathrm{CL}$

cardiolipin

C

curcumin

GMP-CPP

guanosine- $5^{\prime}-[(\alpha, \beta)-$-methyleno]triphosphate

EGCG

epigallocatechin-3-gallate

GTP

guanosine-5'-triphosphate

GUV

giant unilamellar vesicles

LUVs

large unilamellar vesicles 
ZipA soluble protein

YFP

yellow fluorescent protein

\section{Introduction}

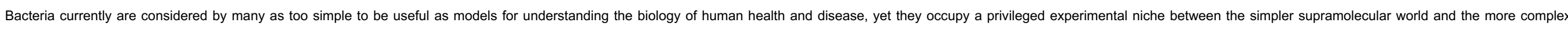

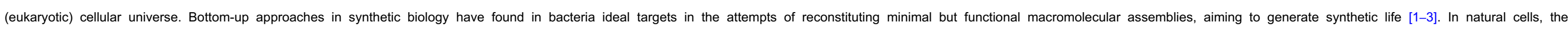

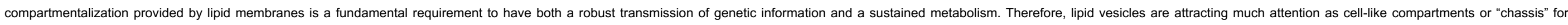
bottom-up synthetic biology $[1,4,5]$.

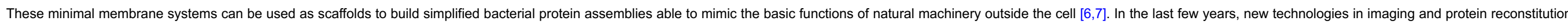
in membranes have put this goal within reach, especially for machineries in which the description of its components is known, and their biochemical properties and their interactions are well characterized [8].

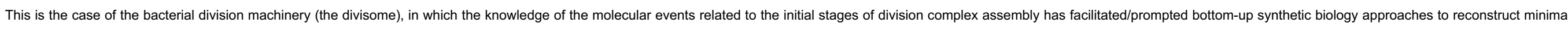

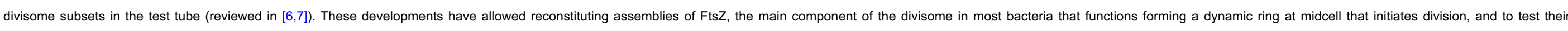

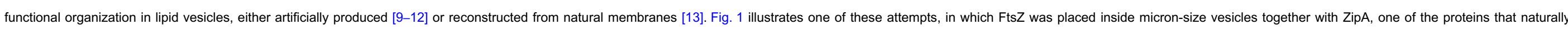

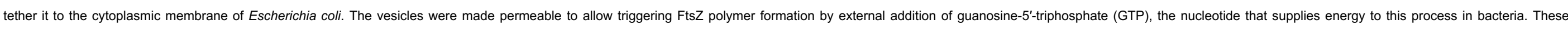
vesicles shrank upon FtsZ polymerization, mimicking to some extent membrane constriction forces in cells [9].
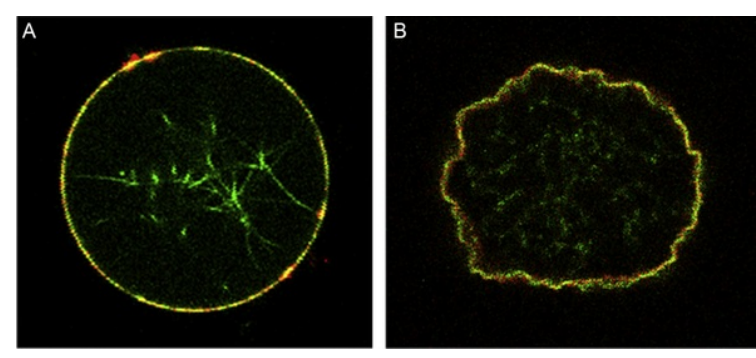

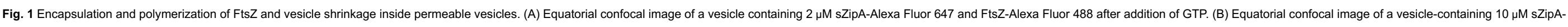
Alexa Fluor 647 with FtsZ-Alexa 488 after addition of GMP-CPP. 


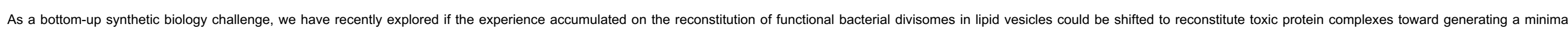

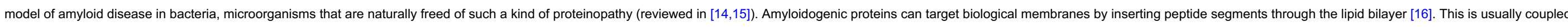

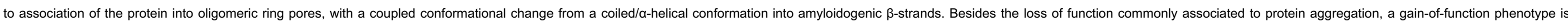

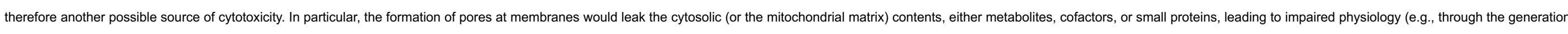

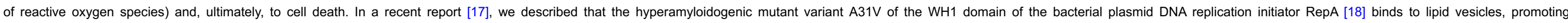

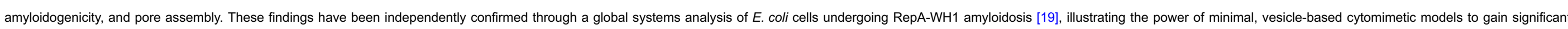

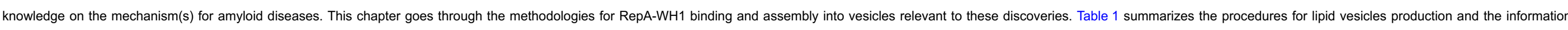
provided by each model system.

Table 1 Summary of the Selected Methods for Vesicles Production Used in This Work

alt-text: Table 1

\begin{tabular}{|c|c|c|c|c|}
\hline & Method & Advantages & Information Provided & References \\
\hline LUVs & Prepared by extrusion techniques & $\begin{array}{l}\text { - Simple } \\
\text { - Quantitative studies of protein-lipid interactions }\end{array}$ & - Binding and interaction of proteins with membranes & {$[17,20]$} \\
\hline \multirow[t]{3}{*}{ GUVs } & Natural swelling in agarose films & $\begin{array}{l}\text { - High yield of vesicles } \\
\text { - Suitable for natural membranes }\end{array}$ & - Membrane binding studies in the absence of oil & {$[17,21-23]$} \\
\hline & Electroformation & $\begin{array}{l}\text { - High yield of vesicles } \\
\text { - Suitable for lipids and natural membranes }\end{array}$ & - Membrane binding studies and incubation with antibodies avoiding the presence of oil & {$[17,21,24,25]$} \\
\hline & Double emulsion method & - High encapsulation efficiency & $\begin{array}{l}\text { - Encapsulation of fluorescent dyes for single vesicle leakage measurements } \\
\text { - Cell-free system encapsulation for protein expression in a biomimetic context }\end{array}$ & {$[9,17,21,26]$} \\
\hline
\end{tabular}

\section{Protein-Lipid Interactions: Experimental Approaches Based on LUVs}

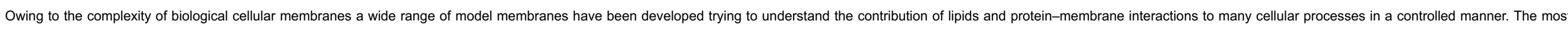

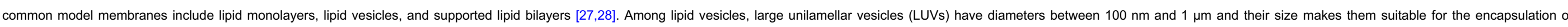

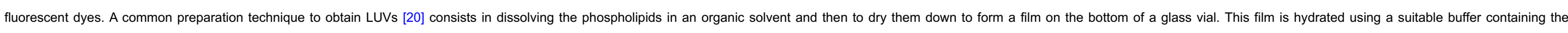

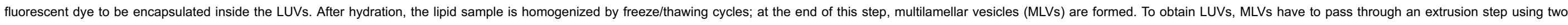

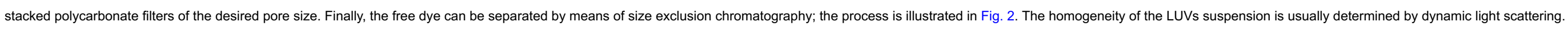




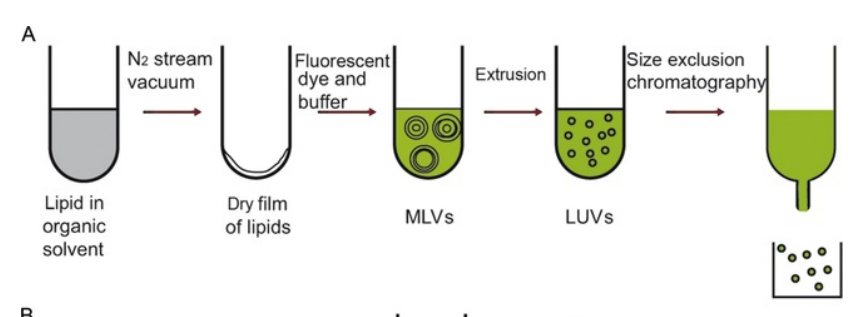

B
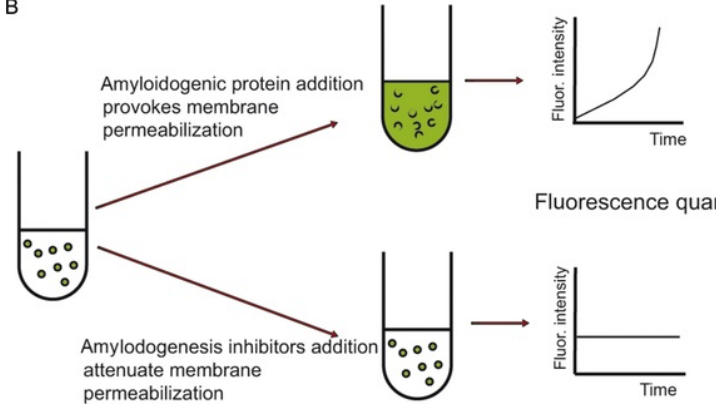

Fig. 2 (A) General strategy for LUVs production and dye encapsulation inside liposomes. (B) An outline of a procedure for monitoring protein (amyloid)-elicited dye release. alt-text: Fig. 2

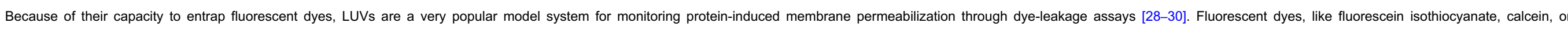

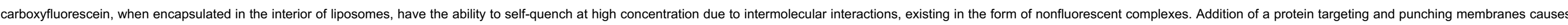
dye dilution in the external medium and consequently an increase in the fluorescent intensity of the dye (Fig. 2B), an event that can be easily monitored in a fluorescence microplate reader [29].

\subsection{Leakage Experiments to Study Protein-Lipid Interactions: The Case of Membrane Targeting by Amyloidogenic RepA-WH1(A31V)}

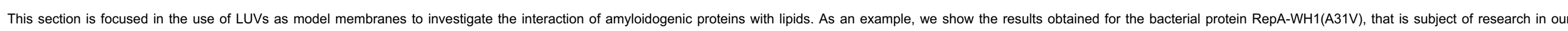

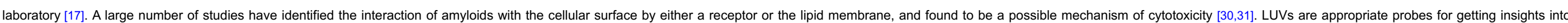

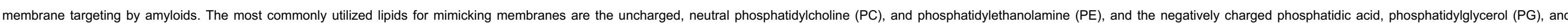

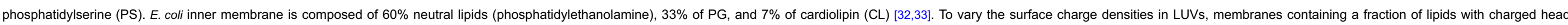
groups like PG or PS are used, supplemented with zwitterionic lipids like PC.

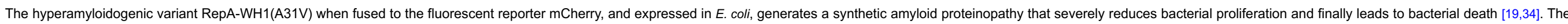

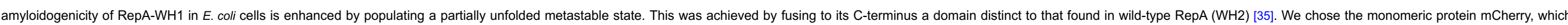

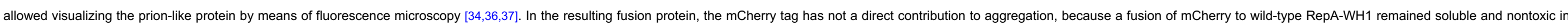
the cytoplasm [34,37].

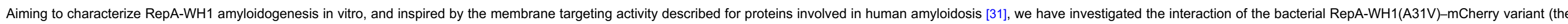

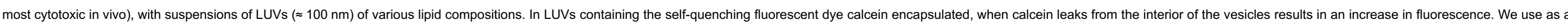
control purified mCherry protein to compare the vesicle-permeabilization effects of both proteins [17,34-37].

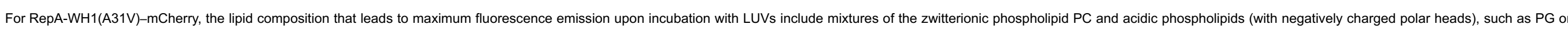

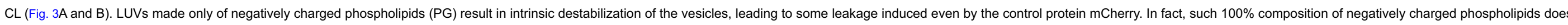

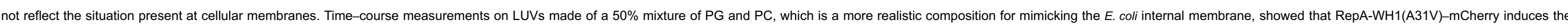

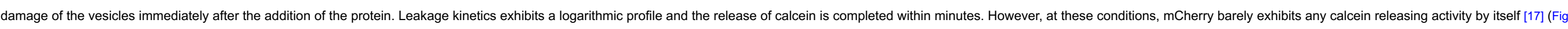




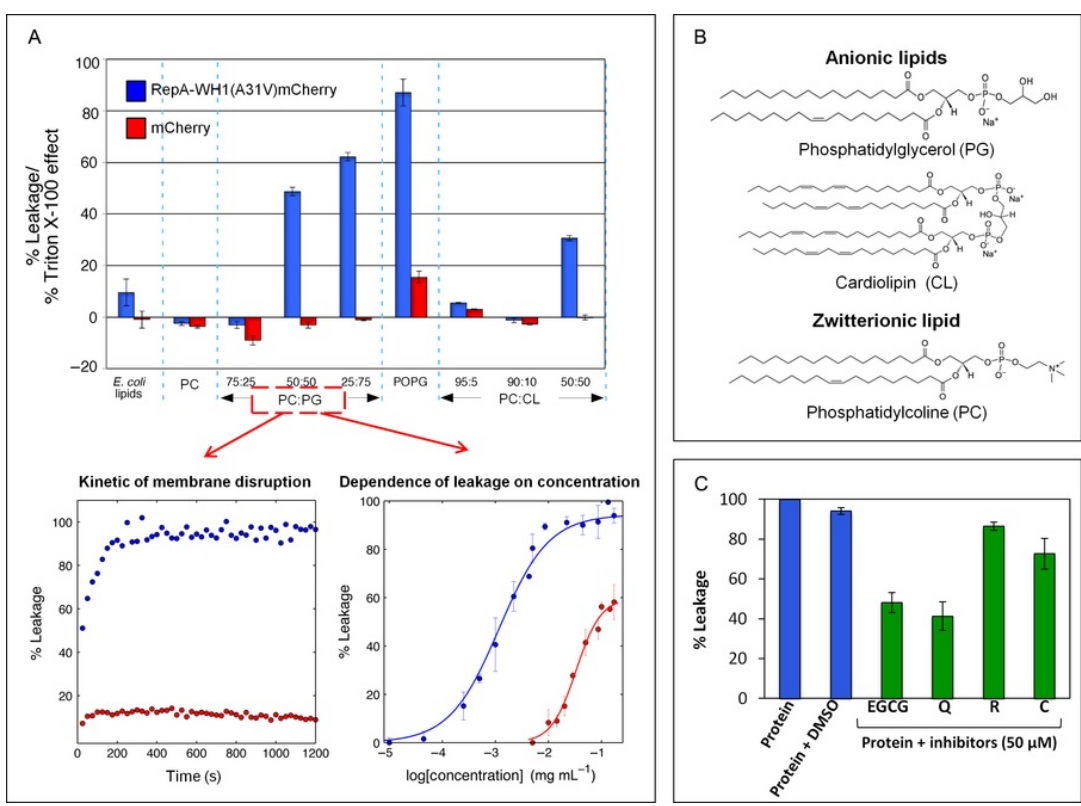

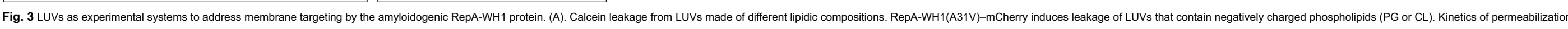

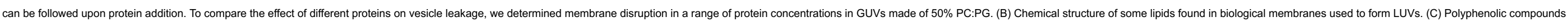
inhibit the interaction of RepA-WH1(A31V)-mCherry with membranes.

Figures were adapted from C. Fernández, R. Núñez-Ramirez, M. Jiménez, G. Rivas, R. Giraldo, RepA-WH1, the agent of an amyloid proteinopathy in bacteria, builds oligomeric pores through lipid vesicles, Sci. Rep. 6 (2016) 23144. alt-text: Fig. 3

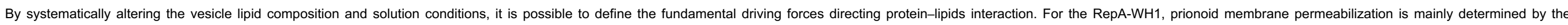

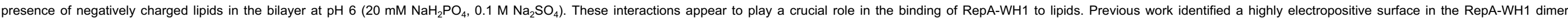

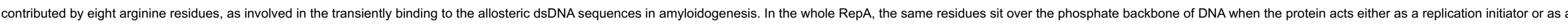
transcriptional repressor. Therefore, it is likely that the arginine patch would be also involved in the electrostatic interaction of RepA-WH1 with the heads of the acidic phospholipids [35,38].

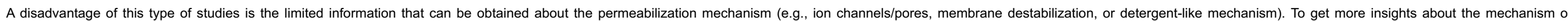
interaction of amyloidogenic proteins with membranes, including single-vesicle measurements, it is necessary to work with additional model systems, as we will show in Section 3 .

\subsection{Leakage Experiments for the Analysis of Amyloidogenesis Inhibitors}

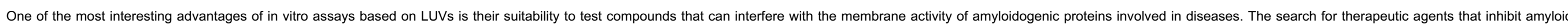

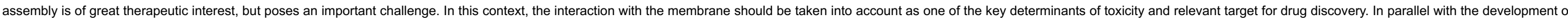

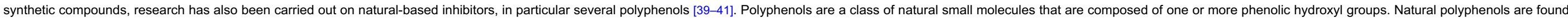
in high concentrations in tea, red wine, different berries, and a wide variety of other natural products from plants.

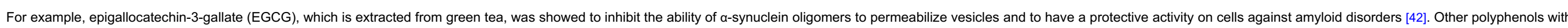
antiamyloidogenic activity include curcumin $(C)$, resveratrol $(R)$, or quercetin $(Q)$. 


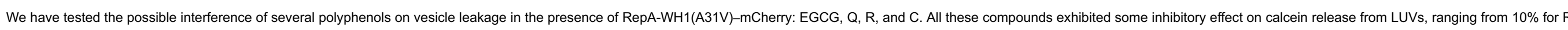

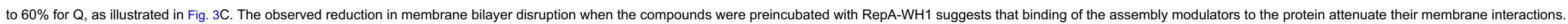

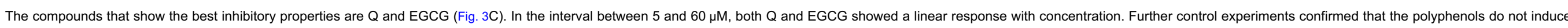
by themselves any detectable dye leakage in absence of protein [17].

\section{Reconstitution of Cytotoxic Bacterial Protein Assemblies in Cell-Like Containers: Experimental Approaches Using Giant Vesicles}

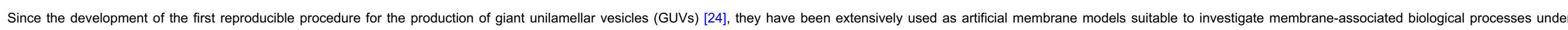

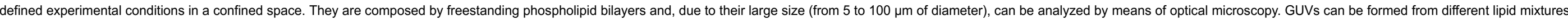
including bacterial membranes [13] and the available strategies for their manipulation allow their use as cell-sized biological reactors and minimal bacterial machineries [21].

\subsection{Preparation of Giant Vesicles}

In this section, we present a short summary of the procedures for preparing GUVs that have been used in the reconstitution studies related to the bacterial prion-like protein RepA-WH1 (Fig. 4).
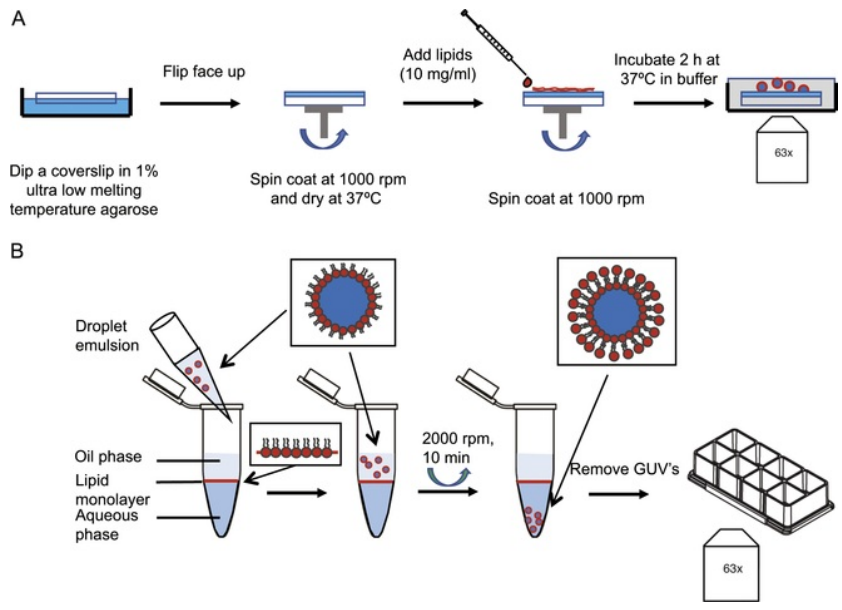

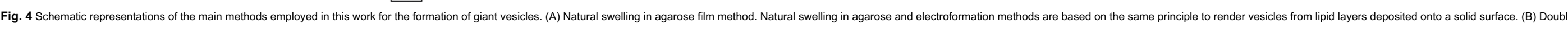
emulsion method. Droplets are coated with the outer lipid monolayer by migration from the oil phase to the aqueous phase by low speed centrifugation.

alt-text: Fig. 4

\subsubsection{Natural Swelling in Agarose Films and Electroformation}

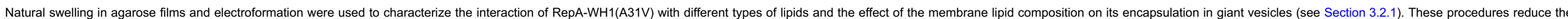
contact with organic solvents to a minimum and are well adapted to reconstitute membrane-associated reactions involving transmembrane proteins and/or native biological membranes.

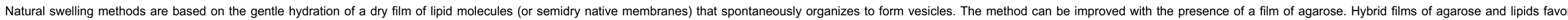
essential factors for vesicles formation (i.e., self-assembly of the lipids into lamellae, separation of lipid lamellae, and liposome fusion to form giant vesicles) [22,23].

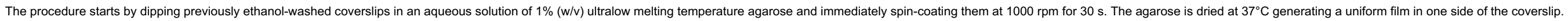

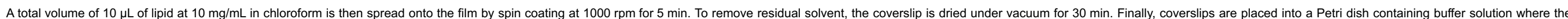
hybrid film is faced upward. Liposomes were generated after incubating for at least $2 \mathrm{~h}$ at $37^{\circ} \mathrm{C}$ (Fig. 4A). 


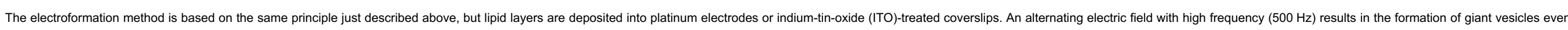

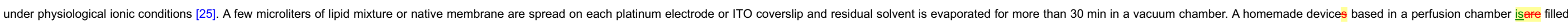
with buffer and giant vesicles are produced in $2 \mathrm{~h}$ at $37^{\circ} \mathrm{C}$ when the electric field is applied. A low frequency $(10 \mathrm{~Hz})$ during $10 \mathrm{~min}$ in the last step can be used for the total separation of the liposomes from the electrodes.

\subsubsection{Double Emulsion Method}

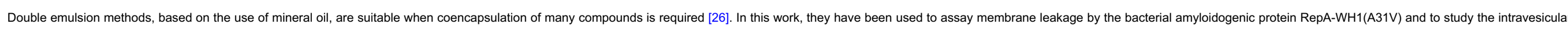

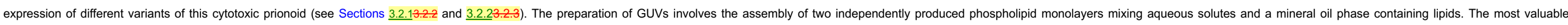
advantage is the rather high encapsulation efficiency and the control on protein entrapment that it provides.

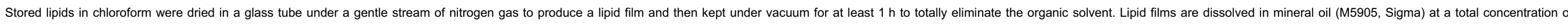

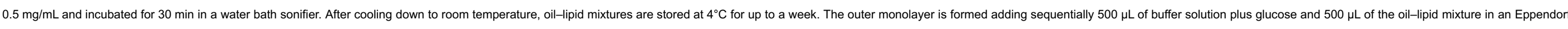

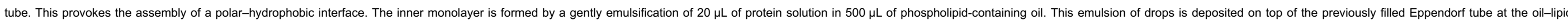

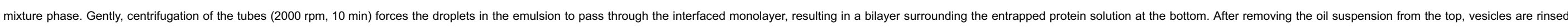
with the buffer to remove oil contaminations and nonencapsulated proteins (Fig. 4B) [9,26].

\subsection{Assembly of a Cytotoxic Amyloidogenic Bacterial Protein Inside GUVs}

\subsubsection{Encapsulation of the Prion-Like Protein RepA-WH1: Effect of Different Lipid Composition and Membrane Leakage Assays}

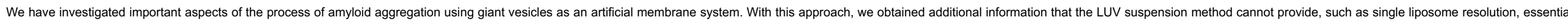
to probe homogeneity in the population of vesicles, or information about the mechanism how the protein exerts damage to membranes $[31,43]$.

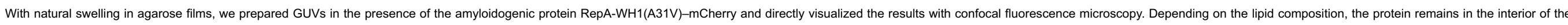

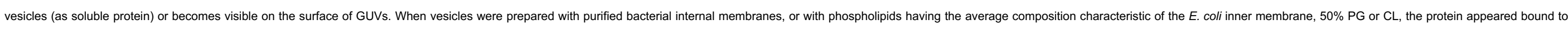
lipids and aggregation at the surface of the vesicle became evident (Fig. 5A). However, RepA-WH1(A31V)-mCherry remained soluble if the lipids are exclusively neutral (PC), whereas the purified mCherry control stayed soluble in all the conditions tested [17]. 


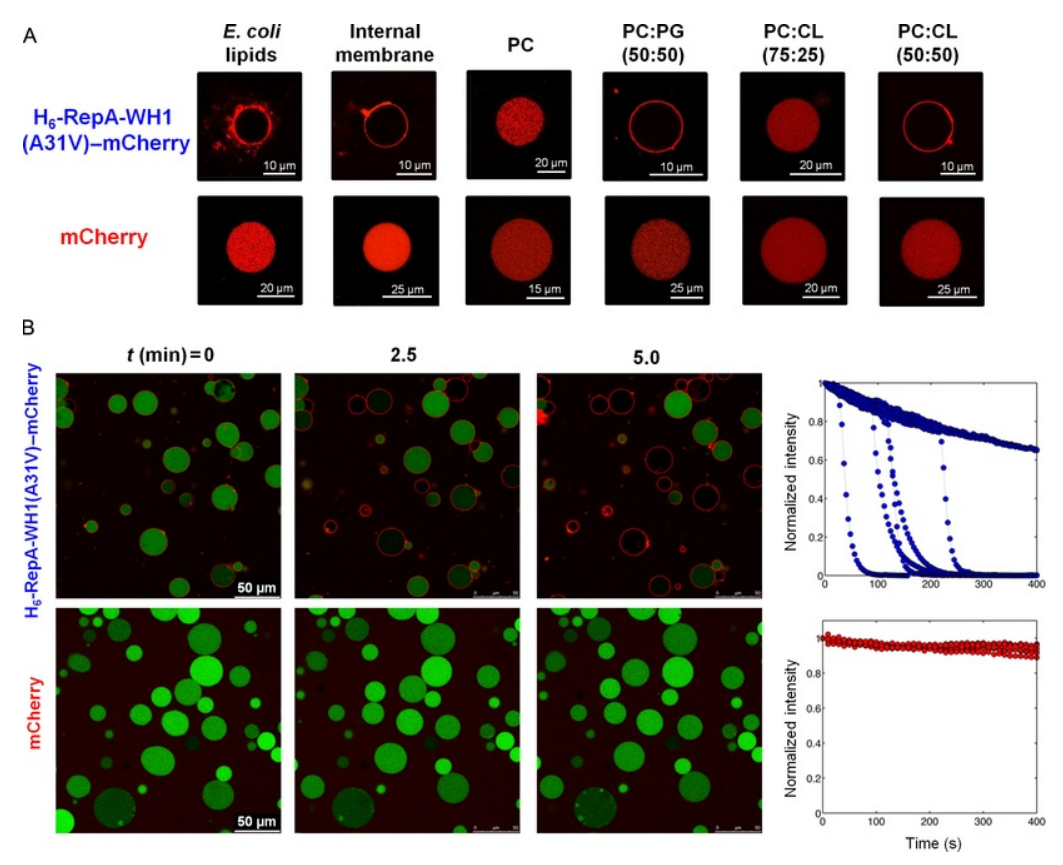

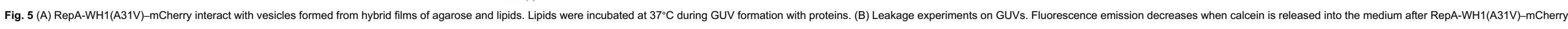
addition (red). GUVs were made of 50\% PC:PG, filled with calcein, using the double emulsion method. The plots on the right show the time course of the change in the normalized fluorescence intensity of several GUVs.

Figure adapted from C. Fernández, R. Núñez-Ramirez, M. Jiménez, G. Rivas, R. Giraldo, RepA-WH1, the agent of an amyloid proteinopathy in bacteria, builds oligomeric pores through lipid vesicles, Sci. Rep. 6 (2016) 23144. alt-text: Fig. 5

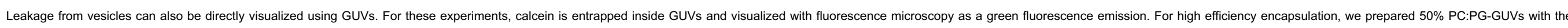

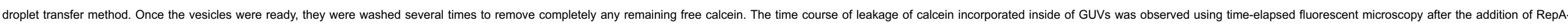

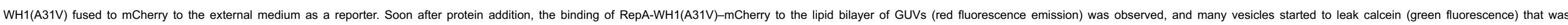

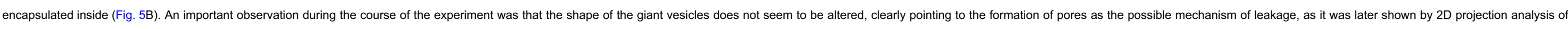
images acquired by electron microscopy [17].

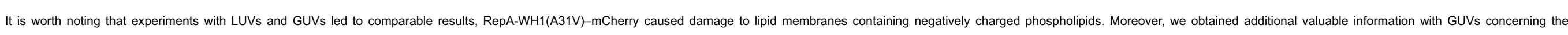
mechanism of leakage.

\subsubsection{Cell Free, Intravesicular Expression of RepA-WH1}

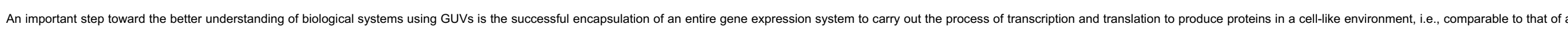
cell. Up to now, the synthesis of functional proteins within liposomes has been examined in several reports using cell-free systems [44-48].

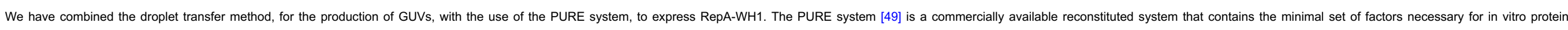

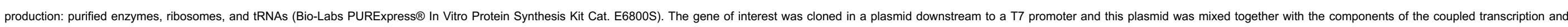
translation machineries. Plasmid and cell-free system were encapsulated inside the vesicles as indicated in Section 3.1.2 and the intravesicular synthesis of the protein proceeds during incubation at $37^{\circ} \mathrm{C}$ (Fig. 6). 

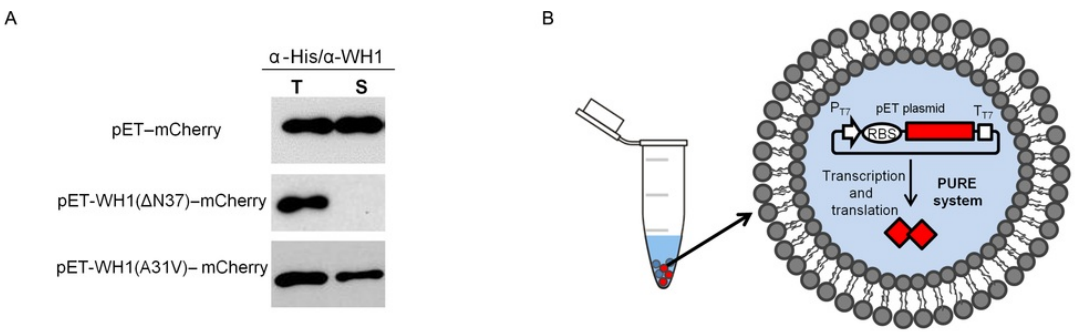

c
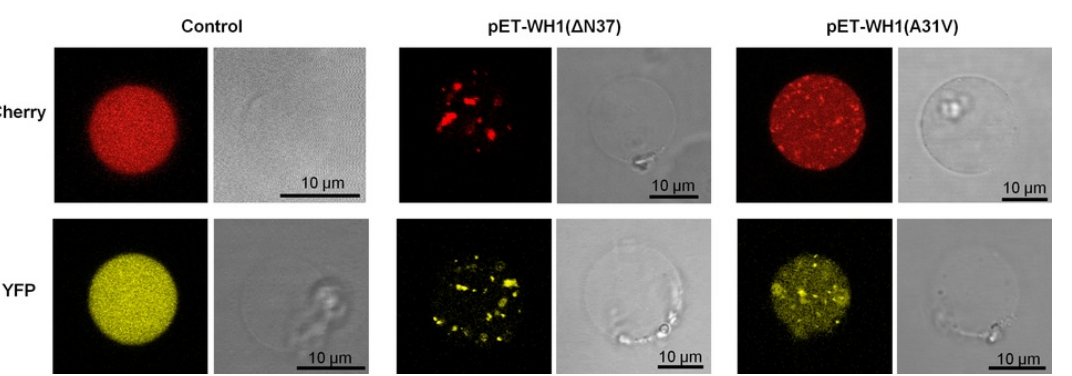

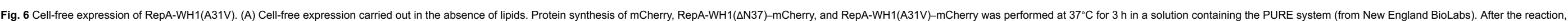

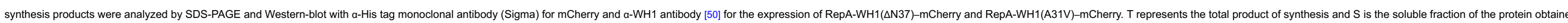

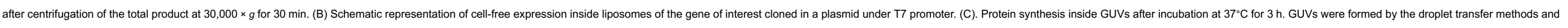
plasmids (encoding RepA-WH1 fusions to mCherry and YFP) and the PURE system were simultaneously encapsulated inside the vesicles. GUVs were observed by confocal microscopy in an 8-well visualization chamber (Lab Tech) alt-text: Fig. 6

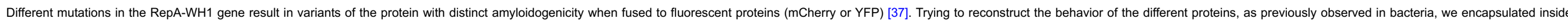

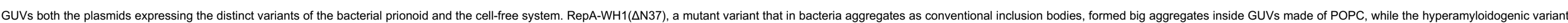

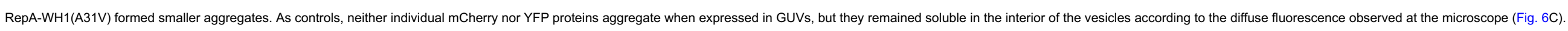

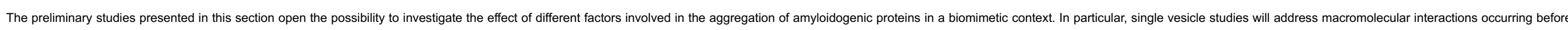
newly synthesized, metastable protein folding intermediates acquire their native conformation, which often lead to the assembly of oligomers on-pathway to the amyloid state

\section{Concluding Remarks}

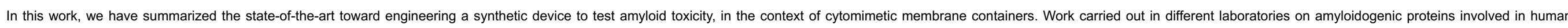

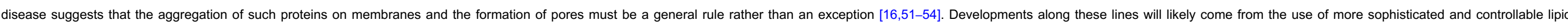

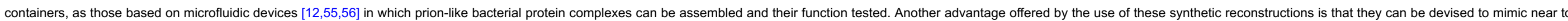

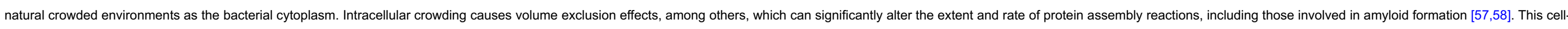

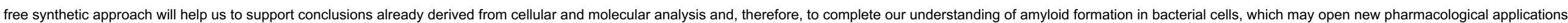

\section{Acknowledgments}

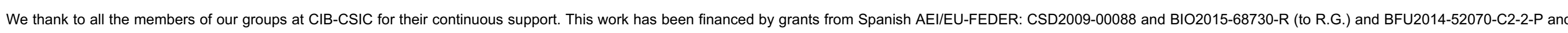
BFU2016-75471-C2-1-P (to G.R.)

\section{References}


[1] C. Chiarabelli, P. Stano, F. Anella, P. Carrara and P.L. Luisi, Approaches to chemical synthetic biology, FEBS Lett. 586, 2012, 2138-2145.

[2] A.P. Liu and D.A. Fletcher, Biology under construction: in vitro reconstitution of cellular function, Nat. Rev. Mol. Cell Biol. 10, 2009, 644-650.

[3] P. Schwille and S. Diez, Synthetic biology of minimal systems, Crit. Rev. Biochem. Mol. Biol. 44, 2009, 223-242.

[4] J.C. Blain and J.W. Szostak, Progress toward synthetic cells, Annu. Rev. Biochem. 83, 2014, 615-640.

[5] F. Caschera and V. Noireaux, Integration of biological parts toward the synthesis of a minimal cell, Curr. Opin. Chem. Biol. 22, 2014, 85-91.

[6] A. Martos, M. Jiménez, G. Rivas and P. Schwille, Towards a bottom-up reconstitution of bacterial cell division, Trends Cell Biol. 22, 2012, 634-643.

[7] G. Rivas, S.K. Vogel and P. Schwille, Reconstitution of cytoskeletal protein assemblies for large-scale membrane transformation, Curr. Opin. Chem. Biol. 22, $2014,18-26$.

[8] T.J. Lagny and P. Bassereau, Bioinspired membrane-based systems for a physical approach of cell organization and dynamics: usefulness and limitations, Interface Focus 5, $2015,20150038$.

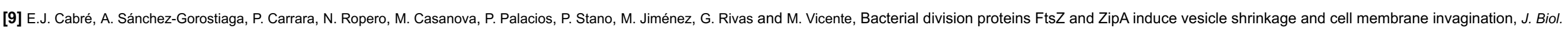
Chem. 288, 2013, 26625-26634.

[10] M. Osawa, D.E. Anderson and H.P. Erickson, Reconstitution of contractile FtsZ rings in liposomes, Science 320, 2008, 792-794.

[11] P. Szwedziak, Q. Wang, T.A.M. Bharat, M. Tsim and J. Löwe, Architecture of the ring formed by the tubulin homologue FtsZ in bacterial cell division, eLife 3, 2014 , e04601.

[12] S. Deshpande, Y. Caspi, A.E.C. Meijerin and C. Dekker, Octanol-assisted liposome assembly on chip, Nat. Commun. 7, 2016, 10447.

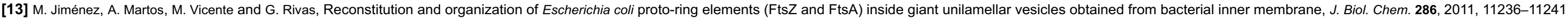

[14] R. Giraldo, S. Moreno-Diaz de la Espina, M.E. Fernández-Tresguerres and F. Gasset-Rosa, RepA-WH1 prionoid. A synthetic amyloid proteinopathy in a minimalist host, Prion 5, 2011, 60-64.

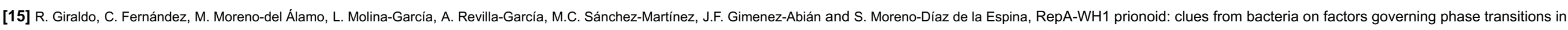
amyloidogenesis, Prion 10, 2016, 41-49.

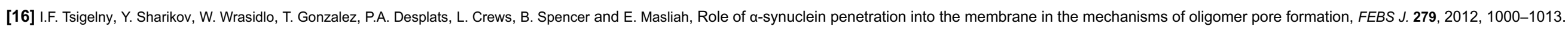

[17] C. Fernández, R. Núñez-Ramírez, M. Jiménez, G. Rivas and R. Giraldo, RepA-WH1, the agent of an amyloid proteinopathy in bacteria, builds oligomeric pores through lipid vesicles, Sci. Rep. 6, 2016, 23144.

[18] R. Giraldo and M.E. Fernández-Tresguerres, 20 years of the pPS10 replicon: insights on the molecular mechanism for the activation of DNA replication in iteron-containing bacterial plasmids, Plasmid 52, $2004,69-83$.

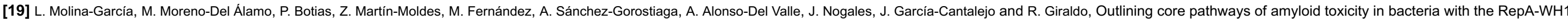
prionoid, Front. Microbiol. 8, 2017, 539.

[20] A. Jesorka and O. Orwar, Liposomes: technologies and analytical applications, Rev. Anal. Chem. 1, 2008, 801-832.

[21] M. Jiménez, A. Martos, E.J. Cabré, A. Raso and G. Rivas, Giant vesicles: a powerful tool to reconstruct bacterial division assemblies in cell-like compartments, Environ. Microbiol. 15, 2013, 3158-3168.

[22] K.S. Horger, D.J. Estes, R. Capone and M. Mayer, Films of agarose enable rapid formation of giant liposomes in solutions of physiologic ionic strength, J. Am. Chem. Soc. 131, 2009, 1810-1811.

[23] A. Weinberger, F.C. Tsai, G.H. Koenderink, T.F. Schmidt, R. Itri, W. Meier, T. Schmatko, A. Schröder and C. Marques, Gel-assisted formation of giant unilamellar vesicles, Biophys. J. 105, 2013 , 154-164.

[24] M.I. Angelova and D.S. Dimitrov, A mechanism of liposome electroformation, Progr. Colloid Polym. Sci. 76, 1988, 59-67.

[25] P. Méléard, L.A. Bagatolli and T. Pott, Giant unilamellar vesicle electroformation from lipid mixtures to native membranes under physiological conditions, Methods Enzymol. $465,2009,161-176$.

[26] S. Pautot, B.J. Frisken and D.A. Weitz, Production of unilamellar vesicles using an inverted emulsion, Langmuir 19, 2003, 2870-2879. 
[27] Y.H. Chan and S.G. Boxer, Model membranes systems and their applications, Curr. Opin. Chem. Biol. 11, 2007, 581-587.

[28] E. Sezgin and P. Schwille, Model membrane platforms to study protein-membrane interactions, Mol. Membr. Biol. 29, 2012, 144-154.

[29] J.M. Rausch and W.C. Wimley, A high-throughput screen for identifying transmembrane pore-forming peptides, Anal. Biochem. 293, 2001, 258-263.

[30] B.D. Van Rooijen, M.M.A.E. Claessens and V. Subramaniam, Membrane permeabilization by oligomeric a-synuclein: in search of the mechanism, PLoS One 5, 2010 , e14292.

[31] H.A. Lashuel and S.M. Butterfiel, Amyloidogenic protein-membrane interactions: mechanistic insight from model systems, Angew. Chem. Int. Ed. 49, $2010,5628-5654$.

[32] S.G. Wilkinson, Gram-negative bacteria, In: C. Ratledge and S.G. Wilkinson, (Eds.), Microbial Lipids, 1988, Academic Press; London, $299-488$.

[33] W.M. O'Leary and S.G. Wilkinson, Gram-positive bacteria, In: C. Ratledge and S.G. Wilkinson, (Eds.), Microbial Lipids, 1988, Academic Press; London, $117-201$.

[34] M.E. Fernández-Tresguerres, S. Moreno-Díaz de la Espina, F. Gasset-Rosa and R. Giraldo, A DNA-promoted amyloid proteinopathy in Escherichia coli, Mol. Microbiol. 77, 2010, 1456-1469.

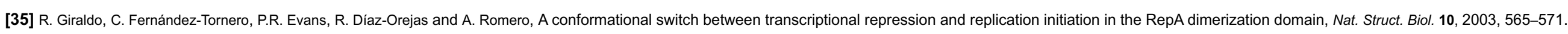

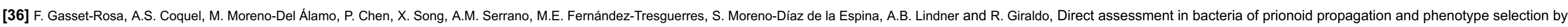
Hsp70 chaperone, Mol. Microbiol. 91, 2014, 1070-1087.

[37] L. Molina-García and R. Giraldo, Aggregation interplay between variants of the RepA-WH1 prionoid in Escherichia coli, J. Bacteriol. 196, $2014,2536-2542$.

[38] F. Gasset-Rosa, M.J. Maté, C. Dávila-Fajardo, J. Bravo and R. Giraldo, Binding of sulphonated indigo derivatives to RepA-WH1 inhibits DNA-induced protein amyloidogenesis, Nucleic Acids Res. 36, 2008, 2249-2256.

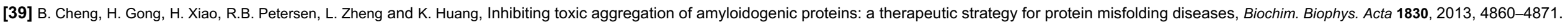

[40] T. Sheynis, A. Friediger, W. Xue, A.L. Hellewell, K.W. Tipping, E.W. Hewitt, S. Radford and R. Jelinek, Aggregation modulators interfere with membrane interactions of b2-microglobulin fibrils, Biophys. J. 105, 2013, 745-755.

[41] R. Malishev, S. Nandi, S. Kolusheva, Y. Levi-Kalisman, F.G. Klärner, T. Schrader, G. Bitan and R. Jelinek, Toxicity inhibitors protect lipid membranes from disruption by Aß42, ACS Chem. Nerosci. 6, $2015,1860-1869$.

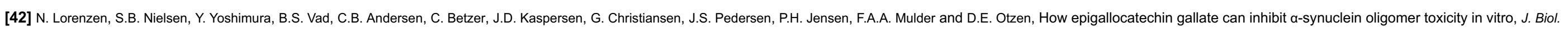
Chem. 289, 2014, 21299-21310.

[43] Y. Tamba and M. Yamazaki, Magainin 2-induced pore formation in the lipid membranes depends on its concentration in the membrane interface, J. Phys. Chem. B 113, $2009,4846-4852$.

[44] H. Saito, Y. Kato, M. Le Berre, A. Yamada, T. Inoue, K. Yosikawa and D. Baigl, Time-resolved tracking of a minimum gene expression system reconstituted in giant liposomes, ChemBioChem 10, 2009, 1640-1643.

[45] P. Stano, Y. Kuruma, T.P. Souza and P.L. Luisi, Biosynthesis of proteins inside liposomes, Methods Mol. Biol. 606, 2010, 127-145.

[46] V. Noireaux and A. Libchaber, A vesicle bioreactor as a step toward an artificial cell assembly, Proc. Natl. Acad. Sci. U.S.A. 10, 2004, 17669-17674.

[47] S.M. Nomura, K. Tsumoto, T. Hamada, K. Akiyoshi, Y. Nakatani and K. Yoshikawa, Gene expression within cell-sized lipid vesicles, ChemBioChem 4, $2003,1172-1175$.

[48] Z. Nourian, W. Roelofsen and C. Danelon, Triggered gene expression in fed-vesicles microreactors with a multifunctional membrane, Angew. Chem. Int. Ed. Engl. 51, 2012, 3114-3118.

[49] Y. Shimizu, A. Inoue, Y. Tomari, T. Suzuki, T. Yokogawa, K. Nishikawa, T. Ueda and C.-f.t.r.w.p. components, Nat. Biotechnol. 19, 2001, 751-755.

[50] M. Moreno-del Álamo, S. Moreno-Díaz de la Espina, M.E. Fernández-Tresguerres and R. Giraldo, Pre-amyloid oligomers of the proteotoxic RepA-WH1 prionoid assemble at the bacterial nucleoid, Sci. Rep. 5, $2015,14669$.

[51] H.A. Lashuel, D. Hartley, B.M. Petre, T. Waltz and P.T.J. Lansbury, Neurodegenerative disease: amyloid pores from pathogenic mutations, Nature 418, $2002,291$.

[52] L. Kjaer, L. Giehm, T. Heimburg and D. Otzen, The influence of vesicle size and composition on alpha-synuclein structure and stability, Biophys. J. 96, 2009, 2857-2870. 
[53] C. Galvagnion, A.K. Buell, G. MeisI, T.C.T. Michaels, M. Vendruscolo, T.P.J. Knowles and C.M. Dobson, Lipid vesicles trigger a-synuclein aggregation by stimulating primary nucleation, Nat. Chem. Biol. 11, 2015 , $229-234$.

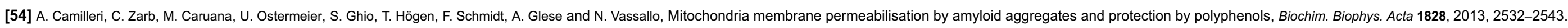

[55] L.R. Arriaga, S.S. Datta, S. Kim, E. Amstad, T.E. Kodger, F. Monroy and D.A. Weitz, Ultrathin shell double emulsion template giant unilamellar lipid vesicles with controlled microdomain formation, Small 10, 2014 , 950-956.

[56] S. Mellouli, B. Monterroso, R. Vutukuri, E. Brinke, V. Chokkalingam, G. Rivas and W.T.S. Huck, Self-organization of the bacterial cell-division protein FtsZ in confined environments, Soft Matter 9, 2013, 10493-10500.

[57] G. Rivas, F. Ferrone, J. Herzfeld and A.P. Minton, Life in a crowded world, EMBO Rep. 5, 2004, 23-27.

[58] A.P. Minton, Implications of macromolecular crowding for protein assembly, Curr. Opin. Struct. Biol. 10, 2000, 34-39.

Keywords: RepA-WH1; Amyloid; GUVs; LUVs; Cell-free synthesis; Synthetic biology

\section{Queries and Answers}

Query:

Please check whether the suggested running head "Assembly and Reconstruction" is appropriate for this chapter.

Answer: The running head is appropriate

Query:

Please check that we have captured all author name and affiliation details correctly. Elsevier affiliation style is Institution, City, State, Country.

Answer: Author names and affiliation details are correct

Query:

Section 3.2.3 is cited in the text but not provided. Please check, and correct if necessary.

Answer: Delete "3.2.2" and "3.2.3" and add "3.2.1" and "3.2.2"

Query:

Please check the edits made to the journal title.

Answer: Checked

Query:

Figure 3, We have received both Color and B\&W figures and both are mismatching. As of now, we are using color figure for web and its converted BW figure for print version. Hope this is fine else please let us know. Answer: We have attached a new B\&W figure

Query:

Please provide better quality figure.

Answer: We have attached new B\&W and color better quality figures

Query:

Figure 5, We have received both Color and B\&W figures and both are mismatching. As of now, we are using color figure for web and its converted BW figure for print version. Hope this is fine else please let us know. 
